\title{
Laparoscopic splenectomy complicated by pancreatic cyst in the course of Hodgkin's disease: case report
}

\author{
Grzegorz Krasowski ${ }^{1,2}$, Andrzej Budzynski ${ }^{3}$, Maciej Miodoński ${ }^{4}$, Katarzyna Seweryn-Serkis ${ }^{5}$, Werner Janus ${ }^{6}$ \\ ${ }^{1}$ Opole University of Technology, Opole, Poland \\ 2Surgical Ward, Krapkowice Health Center, Krapkowice, Poland \\ ${ }^{3} 2^{\text {nd }}$ Chair of Surgery, Jagiellonian University College Medical, Krakow, Poland \\ ${ }^{4}$ Surgical Ward, Provincial Hospital, Opole, Poland \\ ${ }^{5}$ Gynaecological-Maternity Ward, Krapkowice Health Center, Krapkowice, Poland \\ $64^{\text {th }}$ Military Clinical Hospital, Wroclaw, Poland
}

Videosurgery Miniinv 2013; 8 (4): 352-356

DOI: 10.5114/wiitm.2011.35086

\begin{abstract}
This paper describes a case of a patient suffering from Hodgkin's disease and treated by means of minimally invasive surgery: laparoscopic splenectomy. The performance of laparoscopic splenectomy led to a complication in the form of a pancreatic cyst. The cyst was subjected to endoscopic procedures (endoscopic retrograde cholangiopancreatography, with a shaft to the pancreatic tract) and percutaneous drainage. The application of the above methods allowed for a quick introduction of causative treatment. Because in the case in question open surgery methods would impede the introduction of systemic treatment, minimally invasive surgery techniques (laparoscopy and endoscopy) were applied and are discussed below.
\end{abstract}

Key words: laparoscopic splenectomy, endoscopic retrograde cholangiopancreatography, pancreatic cyst, nonHodgkin's lymphoma.

\section{Introduction}

Modern methods of treatment of non-Hodgkin's lymphoma, leukaemia and Hodgkin's disease rely on chemo- and radiotherapy. In some cases, the application of interferon and transplantation of bone marrow are applied. Patients suffering from the abovementioned disorders are subjected to splenectomy if the following symptoms are present: thrombocytopenia, decrease of leukocyte number, splenomegaly with tenderness and pain as a result of recurring spleen infarctions and immune-mediated haemolytic anaemia requiring blood transfusion [1]. The reason for performing splenectomy may also be the uncer- tainty of diagnostic procedures, especially when there is no other possibility to confirm the diagnosis.

Hodgkin's disease itself as well as its treatment lead to immunosuppression of the patient. Therefore, every surgical procedure performed during the course of Hodgkin's disease should be as little invasive as possible. If splenectomy is indicated, laparoscopy is the preferred procedure.

\section{Case report}

At the turn of July and August of 2012, the patient was diagnosed at the Haematology Ward. As spleen lymphoma with associated hepatic and idiopathic 
thrombocytopenia was suspected, the patient was qualified for splenectomy to treat the thrombocytopenia and to confirm the diagnosis. Additionally, the lack of diagnostic peripheral lymph glands and the noncharacteristic view of the bone marrow made it impossible to determine the specific diagnosis.

In view of possible chemotherapy, it was decided to perform the surgery by means of minimally invasive techniques. On 10 September 2012, splenectomy was performed together with perioperative systemic antibiotic therapy.

The patient was positioned on his right side. When ports were introduced, a significantly enlarged spleen was revealed. It was noticed that the basal surface of the organ reached the right lobe of the liver. The splenic flexure of the colon and lienophrenic ligament were incised to make the tail of the pancreas and splenic blood vessels visible. The vessels were clipped and incised in the following order: artery - vein (Photo 1). The above measures were applied to disconnect the organ. Next, by means of a hook electrode, short gastric arteries were closed. An abdominal exploration was performed to look for any auxiliary spleens.

Because of the spleen size, incision of the left anterior auxiliary line was broadened. With the help of manual assistance, the spleen was transferred to a plastic bag and removed. The mass of the removed organ after devascularisation was $3 \mathrm{~kg}$ (Photo 2).During the operation, the condition of the patient was good and no significant bleeding was observed.

However, as the haemoglobin level in the patient's blood was relatively low (HGB $8.5 \mathrm{mg} / \mathrm{dl}$ ), 2 units of the appropriate packed red blood cells (PRBC) were transferred.

On the second day after the surgery, the drain was removed. In the post-operative course, symp-

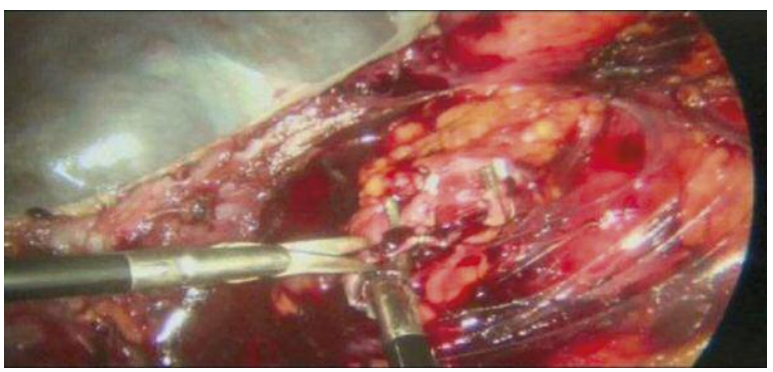

Photo 1. Devascularisation of the spleen (the artery is already clipsed and cut off, vein is clipsed and in the process of cutting off) toms of lung inflammation were observed on the left side with extensive pleural effusion. Conservative treatment was introduced, including systemic empirical antibiotic therapy and targeted antibiotic therapy after microbiological results from the sputum sample were obtained. The lung inflammation symptoms cleared up. On the $10^{\text {th }}$ day after the operation, the patient was discharged in a good general condition and referred to out-patient care. Histopathological and immunohistochemical evaluation confirmed the diagnosis of Hodgkin's lymphoma of mixed cellular type.

While still under the care of the Hematologic Ward, the patient had a PET assay and computed tomography performed. These revealed multifocal localisation of lymphoma in the supra- and subdiaphragmatic lymphatic nodes with bone marrow involvement. Moreover, in the left pleura, a large amount of fluid and a complete collapse of the left lung were detected. In the splenic bed, fluid collections of $20 \mathrm{~cm} \times 20 \mathrm{~cm}$ $\times 15 \mathrm{~cm}$ were detected. These collections, suspected to be an abscess or a pancreatic cyst, were punctured and pancreatic fluid was revealed. Because of the scheduled chemotherapy, a decision to administer a minimally invasive treatment was made.

On 17 October 2012, an endoscopic retrograde cholangiopancreatography (ERCP) was performed. Biliary tracts were contrasted, a shaft was introduced to the pancreatic tract (Photo 3) and a pancreatic cyst was detected in the tail area. However, the damaged area of the pancreatic tract was not found (Photo 4). Next, a prosthesis was established to drain the pancreatic cyst (Photo 5). The patient took these procedures well.

On 22 October 2012, an external drain of the pancreatic cyst was performed by introducing a drain to the fluid collection under ultrasound control (Photo 6). On the first day, $2000 \mathrm{ml}$ of green-white fluid was



Photo 2. Removed spleen 


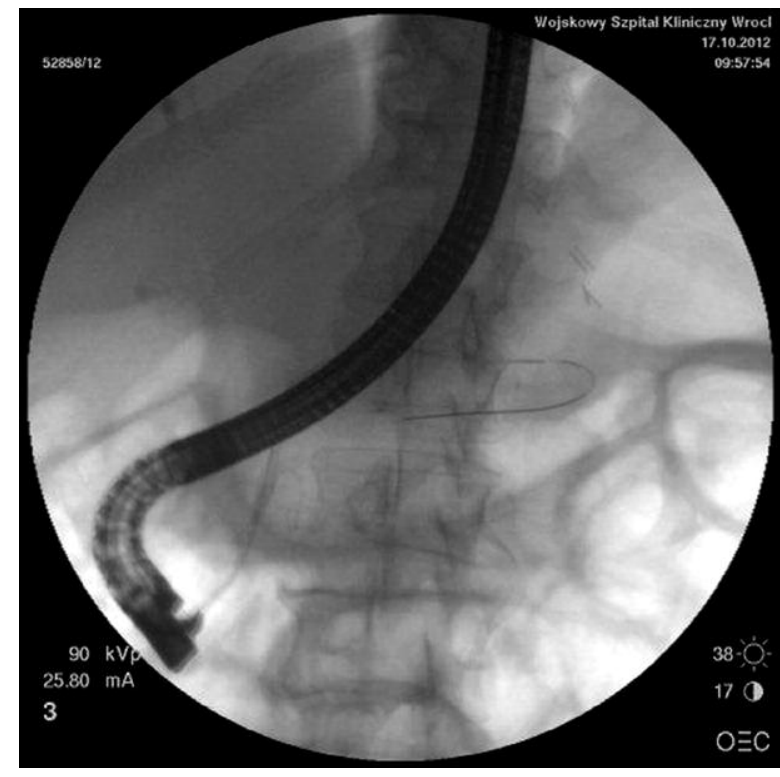

Photo 3. Endoscopic shaft introduced to the pancreatic tract

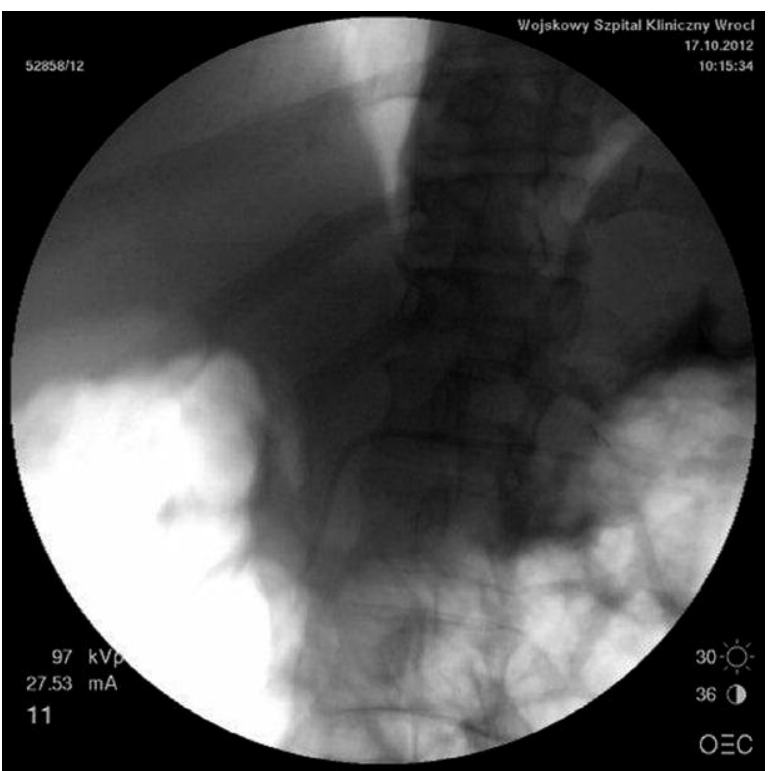

Photo 5. Prosthesis introduced to the pancreatic tract

drained (Photo 7) and ca. $200 \mathrm{ml}$ of fluid was drained on the following days.

As the presence of a non-inflammatory fluid in the left pleura was confirmed by means of radiology (Photo 8), the decision to perform suction drainage of the chest was taken on 23 October 2012. Drainage was established in the $6^{\text {th }}$ intercostal space in the midaxillary line. Initially, 60-300 $\mathrm{ml}$ of bloody effusion fluid was drained off. After that, although the

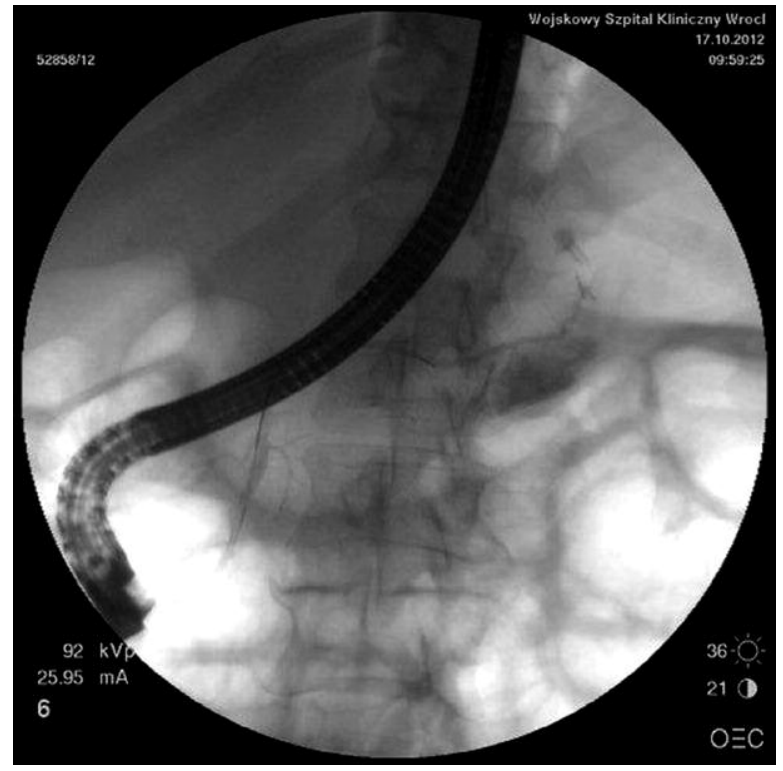

Photo 4. Contrast in the cyst, no damaged area is observed

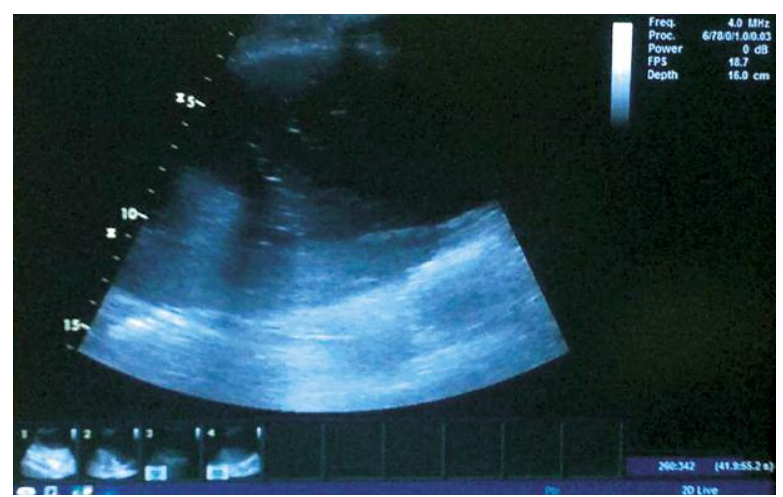

Photo 6. Drain introduced to the cyst under ultrasound control

clinical and radiological symptoms of fluid presence in the pleura did not clear up, no fluid was collected. Therefore, the drain was removed. A new drain was successfully established in the $6^{\text {th }}$ intercostal space in the posterior midaxillary line. After 7 days, the drain was removed. The percutaneous drain of the pancreatic cyst was kept. Fluid excretion reached 2000 $\mathrm{ml} /$ day. A control ultrasound assay did not reveal the presence of the cyst. The patient was discharged in a good general condition with an indication for further out-patient treatment. The patient remained under the care of the Haematology Ward. The percutaneous drain of the cyst was kept during the first cycle of chemotherapy. Excretion of the fluid was ca. $20 \mathrm{ml}$ per day. 




Photo 7. Internal drain of the pancreatic cyst

Presently, the patient is starting a new cycle of chemotherapy because of the underlying disease. A control ultrasound assay of the abdomen did not indicate the presence of fluid collections.

\section{Discussion}

Hodgkin's lymphoma belongs to the group of malignant disorders of the lymphoid tissue. In the course of the disease, the presence of specific cells is observed. The disease develops mainly in the lymphatic nodes. Because the aetiology of the disease is unclear, the risk factors remain not fully recognized. However, it is considered that the risk of occurrence of Hodgkin's lymphoma may be related to an earlier infection caused by the Epstein-Barr virus, responsible for mononucleosis. Also HTLV-I, HTLV-II, HHV-6 viruses (human herpes viruses) and the CMV (cytomegaly) virus are suspected to be potential factors related to Hodgkin's lymphoma development. However, no direct evidence was found to prove this hypothesis. Genetic predispositions related to presence of BCL-2 and TP53 genes are also indicated as possible risk factors. The genetic explanation is strongly backed up with the fact that Hodgkin's lymphoma occurs 10 times more often among relatives than among non-related persons and 100 times more often among monovular twins than among biovular twins. It is estimated that the 5 -year survival rate is between $40 \%$ and $80 \%$ [2].

If lymphoma is suspected and indications for splenectomy exist, a complete splenectomy is performed. Some authors suggest that laparoscopic splenectomy is the procedure of choice [3]. A meta-analysis of 51 trials including 2940 cases of splenectomy revealed a lower incidence of infections in cases where laparoscopic splenectomy was performed as opposed to open surgery splenectomy [4].

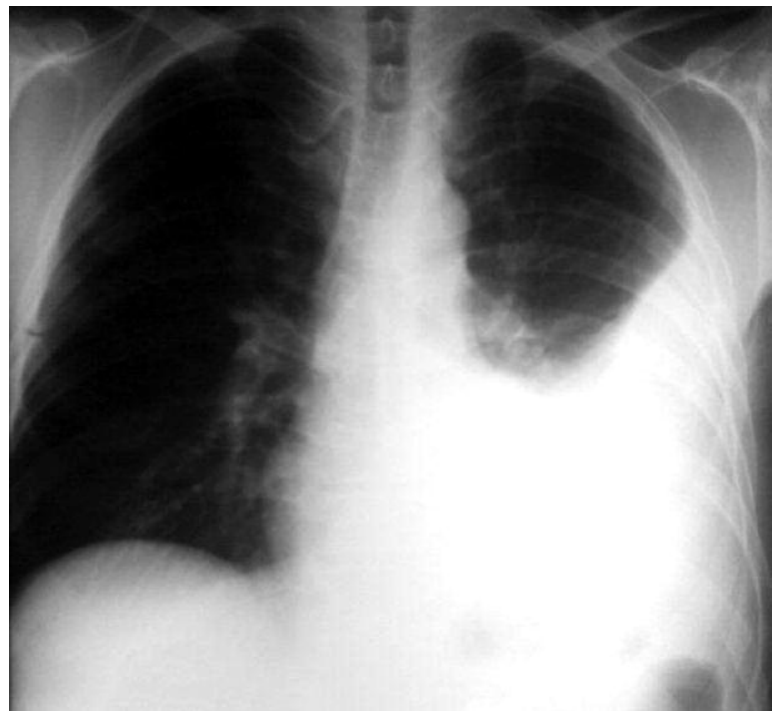

Photo 8. Fluid in the left pleura

Before splenectomy, every patient should be submitted to procedures decreasing the chance of infection. Vaccination against capsular bacteria is one of the means to achieve it. According to recommendations, laparoscopic splenectomy should be performed in specialized medical centres. It not only increases the quality of the surgery performed, but also allows the development of rules to prevent complications [5]. However, if these occur, a specialized centre offers more possibilities of early detection and introduction of appropriate measures. During the course of the surgery, the patient is placed on his right side or slightly slantwise with the left side elevated. This last position is especially favourable in the case of large spleens. In this position, the anatomical structures of the splenic hilum are more visible. In case of pneumoperitoneum formation by means of closure techniques (using a Veress needle), it is important to introduce the insufflation needle in the highest point of the abdomen, i.e. in the patient's anterior axillary line, if he lies on his side. The preferred operative technique is the devascularisation procedure consisting in visualization, closure and cutting the splenic veins off. Deviations from this method are possible, particularly if an endostapler or LigaSure device is used. These methods are very efficient in the case of partial resections. Another technique that may be used is the semi-open method utilizing a special sleeve, in which the hand of the surgeon replaces one of the tools. This method is also applied in cases of large spleens [3]. 
In the present case, the splenic blood vessels were revealed after the release of the lieno-colonic and lienophrenic ligament and pancreatic tail revelation. The pancreatic cyst probably formed as a complication of splenectomy. The authors suspect that during the dissection of the spleen or operation on the blood vessels, thermal or mechanical destruction of the pancreatic tail parenchyma took place. It led to the inflammation and the resulting cyst formation. Another potential reason for occurrence of the cyst may be the lack of vascular supply, when vessels are clipped far from the spleen hilus, which took place in this situation as well.

Complications of laparoscopic splenectomy may occur both intraoperatively and postoperatively or be a result of the underlying disease.

During the surgery, the surgeon may face difficulties associated mostly with bleeding from large blood vessels such as the spleen artery or small blood vessels (short gastric arteries).

It is also possible to harm the organs situated close to the spleen such as the stomach or colon (risk of perforation) or pancreas (inflammatory state after thermal or mechanical destruction).

The most important postoperative complications are bleeding, thrombosis-related complications and late-onset sepsis. Therefore, haemostasis control during the surgery, and perioperative anti-thrombotic and antibiotic prophylaxis are of paramount importance [6]. The need for a routine abdomen drain after splenectomy is still disputed. However, in the present case, such a drain was left in the splenic bed as gastric vessels were secured only by a hook electrode [7].

There are cases of effusion fluids in the left pleura after surgery in the upper segment of the abdomen [8]. However, this symptom may be related to a compromised immune system of the patient. In the present case, the patient was subjected to perioperative antibiotic therapy, but its application does not always guarantee a lack of infective complications after splenectomy [9]. Similar, local antibiotic prophylaxis using a gentamicin surgical implant may not guarantee a lack of postoperative infections [10].

Percutaneous drainage, endoscopic drainage and operative treatment are the acknowledged methods of pancreatic cyst treatment. Trials have proven that false cysts need to communicate with the pancreatic tract [11]. In the present case, we decided not to use an operative internal drain. The reason was a high risk of occurrence of primary or secondary anasto- mosis dehiscence during the planned chemotherapy. In the case of a cyst of such size, single percutaneous drainage might not bring stable effects. The application of only ERCP and the introduction of a prosthesis to the pancreatic tract might also not bring the desired effects. Therefore, we decided to combine the above-mentioned methods and to perform ERCP and the endoscopic drainage first, followed by a percutaneous drain.

\section{Conclusions}

The large size of the spleen is not an absolute contraindication for the application of laparoscopic splenectomy. However, due to the spleen size, some technical difficulties may arise. Minimally invasive techniques may be successfully applied for splenectomy and for the treatment of some postoperative complications. During pancreatic cyst surgery, good results may be achieved by the application of both internal and external drainage.

\section{References}

1. Scharf R, Ziemski JM. Splenectomy in hematological diseases. In: Surgery. Szmidt J, Kużdżał J (eds.). Medycyna Praktyczna, Krakow 2010; 1083-8.

2. Meder J. Ziarnica złośliwa. In: Choroby wewnętrzne [Polish]. Szczeklik A (ed.). Medycyna Praktyczna, Krakow 2006; 1531-7.

3. Budzyński A. Laparoscopic splenectomy. In: Surgery. Szmidt J, Kużdżał J (eds.). Medycyna Praktyczna, Krakow 2010; 1096-103.

4. Głowacki J, Stefaniak T, Gruca Z. Surgical site infection in laparoscopic and endoscopic surgery. Videosurgery Miniinv 2008; 3 : 10-6.

5. Maccabee D, Hunter JG. Laparoscopic splenectomy. In: Current surgical therapy. Cameron JL (ed.). Philadephia, Mosby 2008; 1341-5.

6. Scharf R, Szczepanik A, Ziemski JM. Postoperative complications of splenectomy. In: Surgery. Szmidt J, Kużdżał J (eds.). Medycyna Praktyczna, Krakow 2010; 1103-5.

7. Major P, Matłok M, Pędziwiatr M, Budzyński A. Do we really need routine drainage after laparoscopic adrenalectomy and splenectomy? Videosurgery Miniinv 2012; 7: 33-9.

8. Kołomecki K, Cywiński J, Bartnicki J, et al. Hydrothorax as a complication of upper abdominal surgery Videosurgery Miniinv 2006; 2: 59-64.

9. Budzyński A. Risk of infection after splenectomy, source of hemocidins, antiseptic polypeptids. Postdoctoral dissertation. Medycyna Praktyczna, Krakow 2007

10. Migaczewski M, Zub-Pokrowiecka A, Budzyński P, et al. Prevention of early infective complications after laparoscopic splenectomy with the Garamycin sponge. Videosurgery Miniinv 2012; 8: 105-10.

11. Lampe P, Mrowiec S. Pancreatic cysts. In: Burgery. Szmidt J, Kużdżał J (eds.). Medycyna Praktyczna, Krakow 2010; 1036-44.

Received: 7.03.2013, accepted: 19.03.2013. 